\title{
Abordagens teóricas em dados governamentais abertos
}

Lucas Geraldo Campos Dalenogare

Mestrando em Administração no Departamento de Ciências Administrativas da UFRN. Especialista em Gestão Pública. Graduado em Administração.

lucascdalenogare@gmail.com

Maria Arlete Duarte de Araújo

Doutora em Administração. Professora titular da Universidade Federal do Rio Grande do Norte.

Departamento de Ciências Administrativas.

mariaarlete1956@gmail.com

Editor Científico: José Edson Lara

Organização Comitê Científico

Double Blind Review pelo SEER/OJS

Recebido em 22.06.2018

Aprovado em 12.03.2019

Este trabalho foi licenciado com uma Licença Creative Commons - Atribuição - Não Comercial 3.0 Brasil 


\title{
Resumo
}

Objetivo do estudo: Este artigo tem por objetivo identificar as abordagens teóricas adotadas internacionalmente, nos últimos cinco anos, em estudos sobre Dados Governamentais Abertos (DGA).

Metodologia/abordagem: Optou-se pela realização de uma Revisão Sistemática de Literatura (RSA), guiada pelo protocolo Kitchenham (2007), com o suporte da ferramenta ATLAS.ti. As análises abrangeram vinte e sete estudos realizados em dezenove países.

Originalidade/relevância: A relevância teórica do trabalho reflete na identificação dos aportes teóricos de sustentação das pesquisas sobre DGA e na verificação da presença dos elementos para a constituição de uma escola de pensamento sobre o tema.

Principais resultados: Dos estudos analisados emerge a discussão de elementos teóricos acerca dos próprios dados abertos - como conceitos, requisitos, barreiras e benefícios - e de teorias associadas - como Teoria Institucional, Teoria Cognitiva, Teoria do Estado Informacional, Teoria de Ecossistemas, Teorias de Informação Interagência, da Perspectiva Multi-Nível e de Cross-boundary ou Interorganizacional, sinalizando para a ausência de uma escola de pensamento que aglutine os estudiosos do tema. Outrossim, revela-se também o potencial das políticas de dados abertos para um maior fortalecimento da democracia, geração de valor econômico, inovações e avanços técnico-gerenciais nas organizações públicas.

Contribuições teóricas/metodológicas: A principal contribuição teórica se traduz no conhecimento das principais abordagens teóricas associadas a DGA, e a contribuição metodológica no rigor do desenvolvimento de um protocolo de RSA e da própria análise temática com suporte de um software de análise qualitativa.

Contribuições sociais/gerenciais: A contribuição social remete ao levantamento de benefícios e barreiras das iniciativas em DGA, que se explorados podem levar a uma maior aproximação entre governos e cidadãos.

Palavras-chave: Dados governamentais abertos. Dados públicos. Política de dados abertos.

\section{Theoretical approaches in open government data}

\begin{abstract}
Objective of the study: This article aims to identify the theoretical approaches adopted internationally in the last five years in Open Government Data (DGA) studies.

Methodology/approach: We decided to perform a Systematic Literature Review (RSA), guided by the Kitchenham protocol (2007), supported by the ATLAS.ti tool. The analyzes covered twenty-seven studies conducted in nineteen countries.
\end{abstract}


Originality/relevance: The theoretical relevance of the work reflects the identification of the theoretical support of research on DGA and the verification of the presence of the elements for the constitution of a school of thought on the subject.

Main results: From the analyzed studies emerges the discussion of theoretical elements about the open data itself - such as concepts, requirements, barriers and benefits - and associated theories - such as Institutional Theory, Cognitive Theory, Informational State Theory, Ecosystem Theory, Interagency Information, from the Multi-Level Perspective and Crossboundary or Interorganizational, signaling the absence of a school of thought that brings together scholars of the subject. Moreover, the potential of open data policies to further strengthen democracy, generate economic value, innovations and technical-managerial advances in public organizations is also revealed.

Theoretical/methodological contributions: The main theoretical contribution is the knowledge of the main theoretical approaches associated with DGA, and the methodological contribution to the rigorous development of an RSA protocol and thematic analysis itself supported by qualitative analysis software.

Social/management contributions: Social contribution refers to the survey of benefits and barriers of initiatives in DGA, which if explored can lead to a closer relationship between governments and citizens.

Keywords: Open government data. Public data. Open data policy.

\section{Enfoques teóricos en datos gubernamentales abiertos}

\section{Resumen}

Objetivo del estudio: Este artículo tiene como objetivo identificar los enfoques teóricos adoptados internacionalmente en los últimos cinco años en estudios de datos de gobierno abierto (DGA).

Metodología/enfoque: Decidimos realizar una Revisión sistemática de literatura (RSA), guiada por el protocolo Kitchenham (2007), con el apoyo de la herramienta ATLAS.ti. Los análisis abarcaron veintisiete estudios realizados en diecinueve países.

Originalidad/relevancia: La relevancia teórica del trabajo refleja la identificación del soporte teórico de la investigación sobre DGA y la verificación de la presencia de los elementos para la constitución de una escuela de pensamiento sobre el tema.

Resultados principales: De los estudios analizados surge la discusión de elementos teóricos sobre los datos abiertos en sí mismos, como conceptos, requisitos, barreras y beneficios, y teorías asociadas, como la teoría institucional, la teoría cognitiva, la teoría del estado informacional, la teoría del ecosistema, La información interagencial, desde la perspectiva multinivel y transfronteriza o interorganizacional, indica la ausencia de una escuela de pensamiento que reúna a los estudiosos de la materia. Además, también se revela el potencial 
de las políticas de datos abiertos para fortalecer aún más la democracia, generar valor económico, innovaciones y avances técnico-gerenciales en las organizaciones públicas.

Contribuciones teóricas/metodológicas: La principal contribución teórica es el conocimiento de los principales enfoques teóricos asociados con DGA, y la contribución metodológica al desarrollo riguroso de un protocolo RSA y análisis temático en sí respaldado por un software de análisis cualitativo.

Contribuciones sociales/de gestión: La contribución social se refiere a la encuesta de beneficios y barreras de las iniciativas en DGA, que si se explora puede conducir a una relación más estrecha entre los gobiernos y los ciudadanos.

Palabras clave: Datos gubernamentales abiertos. Datos públicos. Política de datos abiertos.

\section{Introdução}

As organizações públicas estão constantemente sujeitas a demandas por parte dos cidadãos. Predominantemente, essas manifestações estão relacionadas à qualidade e entrega dos serviços prestados, maior transparência e participação na formulação, acompanhamento e avaliação das políticas públicas e maior responsabilização dos gestores sobre a utilização dos recursos públicos. As demandas emergem em cenários econômicos, políticos e sociais mutáveis e são potencializadas pela crescente utilização de novas tecnologias (Albano, 2014), assim como pelo aumento crescente de novas formas de acesso à informação e ampliação da escolarização, que podem impactar diretamente a relação governo-sociedade.

Neste contexto, como apontam Yang e Wu (2016), a temática de Dados Governamentais Abertos (Open Government Data) tem chamado tanto a atenção de estudiosos como dos setores público e privado, em todo o mundo, visto que pode contribuir para o fortalecimento da transparência e da responsabilização dos governos, na medida em que oportuniza o acesso dos cidadãos ao conjunto de dados produzidos na esfera pública e, por conseguinte, cria condições para a geração de valor público, fortalecendo os laços do tecido social.

Wang e Lo (2015), contudo, indicam que boa parte das iniciativas dos governos sobre abertura de dados governamentais ainda se encontra em estágio inicial, existindo poucas pesquisas sobre a execução das políticas e seus impactos. Ressaltam ainda que essas iniciativas são acompanhadas por vários impedimentos e barreiras, relacionadas a limitações na 
disponibilização e uso de dados e em relação ao próprio desenvolvimento das referidas políticas.

Na visão de Corrêa, Paula, Corrêa e Silva (2017), o movimento em torno da abertura de dados governamentais ganhou força com o estabelecimento de uma série de princípios técnicos e conceituais para guiar a abertura de registros públicos através da internet, com a finalidade de tornar os dados públicos livremente utilizáveis, reutilizáveis e redistribuíveis a qualquer indivíduo.

O aumento do apelo pela disponibilização de dados públicos, pelos entes governamentais, tem exigido dos governos nacionais e locais respostas sobre diversas questões relacionadas a dados abertos (Conradie \& Choenni, 2014). As respostas, no entanto, apresentam certa imaturidade sobre os requisitos da disponibilização de dados, especialmente sobre como divulgá-los corretamente, de modo a contribuir para o fortalecimento de uma cultura em torno da matéria (Corrêa et al., 2017). Além disso, as políticas desenvolvidas nesse sentido são recentes e seus efeitos ainda pouco avaliados.

Assim sendo, torna-se importante conhecer e explorar os estudos desenvolvidos com o tema Dados Governamentais Abertos - OGD e encontrar respostas para as seguintes questões: quais abordagens teóricas têm sido adotadas para fundamentar os estudos em torno dos dados abertos? O que evidenciam os resultados dos estudos? Quais os avanços e limites da discussão sobre dados abertos?

Para dar conta dessa problemática e considerando o debate recente sobre o assunto, optou-se por realizar uma Revisão Sistemática de Literatura (RSL), adotando-se o Protocolo Kitchenham (2007), versão 2.3, que estabelece diretrizes para a realização de pesquisas nesse formato na área de Engenharia de Software, mas com possível aplicação em áreas diversas, com o objetivo de identificar e analisar o "estado da arte" sobre Dados Governamentais Abertos OGD no contexto internacional de produção científica das áreas de Administração e Administração Pública.

As seções que seguem apresentam, além dessa Introdução, a descrição detalhada dos procedimentos metodológicos adotados; a análise dos dados, com informações em nível de metadados (análise descritiva); aspectos teóricos relevantes à pesquisa (análise temática); análise comparativa entre os trabalhos dos países que mais publicam sobre o tema; e uma reflexão sobre a produção analisada, nas considerações finais, para argumentar que ainda não se tem uma escola de pensamento que aglutine os estudiosos do tema. 


\section{Metodologia}

Uma revisão sistemática de literatura é um meio de identificar, avaliar e interpretar todas as pesquisas relevantes a uma pergunta de pesquisa em particular, ou tópico de alguma área, ou fenômeno de interesse. Os estudos individuais que contribuem com uma revisão sistemática são chamados de estudos primários; já uma revisão sistemática é uma forma secundária de estudo (Kitchenham, 2007).

As bases de pesquisa utilizadas para a realização das buscas dos estudos primários foram ScienceDirect (Elsevier) e SCOPUS (Elsevier), tendo sido SCOPUS acessada através do recurso remoto e restrito CAFE da Plataforma de Periódicos CAPES (BR). As palavras-chave foram "dados", "abertos" e "governamentais". E finalmente, a string de busca assim se constituiu: "(in title) "dados" OR (in title) "data" AND (in title) "abertos" OR (in title) "open" AND (in title) "governamentais" OR (in title) "government"; (com as expressões entre aspas e entre parênteses de um lado e de outro dos conetivos "AND")".

Como critérios de inclusão foram considerados: estudos (artigos) publicados dentro do espaço temporal de cinco anos - 2013 a 2017; áreas de Administração e Administração Pública; disponíveis em português ou inglês; classificação dos periódicos nacionais no ano da publicação igual ou superior a B2 no Qualis Capes; Fator de Impacto JCR dos periódicos internacionais igual ou acima da média da categoria no ano da publicação; e relação com o tema de pesquisa a partir da análise do título e resumo.

Somados os resultados de ambas as bases de pesquisa, 118 estudos foram encontrados, sendo 90 na base SCOPUS e 28 na base ScienceDirect.

Com a aplicação dos critérios de inclusão e exclusão o corpo final de estudos resultou em 27, visto que na base ScienceDirect foram excluídos 12 artigos, restando 16. Por sua vez, na base SCOPUS foram excluídos 79 artigos, restando 11. Os estudos finais selecionados para a RSL foram armazenados no gerenciador de arquivos Mendeley Desktop.

No que se refere à análise e síntese dos dados, as mesmas foram realizadas a partir da leitura integral dos estudos selecionados e seguiram a sistematização abordada por Sampiéri, Collado e Lúcio (2013), que estabelece a análise aberta ou em primeiro plano, com a geração de códigos ou unidades de significado; análise axial ou em segundo plano, com a geração de categorias e identificação da relação entre elas; e análise seletiva ou temática e interpretação, 
onde são constituídos os temas, proposições, teorias ou explicações que buscam atender ao objetivo da pesquisa, bem como responder às indagações iniciais definidas.

Enfim, o processo de análise contou com a utilização do software de análise qualitativa de dados ATLAS.ti, versão 7.5.7.

\section{Análise dos dados}

\subsection{Análise Descritiva}

Após a aplicação dos critérios de inclusão e exclusão dos estudos selecionados, restaram 27 artigos cujas principais informações são apresentadas, de forma sintetizada, a seguir:

a) Nacionalidade: os estudos alcançaram um total de 38 instituições afiliadas em 19 países, conforme detalhamento da Tabela 1;

\section{Tabela 1}

Países de Origem

\begin{tabular}{|c|c|}
\hline Países de Origem & Artigos Vinculados \\
\hline Alemanha & $A 4, A 25$ \\
\hline Austrália & $A 2, A 5$ \\
\hline Bélgica & A14 \\
\hline Brasil & $A 18, A 22$ \\
\hline Chile & A19 \\
\hline China & A9 \\
\hline Estados Unidos & $A 2, A 3, A 5, A 8, A 10, A 19, A 27$ \\
\hline Gana & $A 12$ \\
\hline Irlanda & $A 13$ \\
\hline Itália & $A 11, A 24$ \\
\hline Países Baixos & $A 14, A 17, A 20, A 23$ \\
\hline Portugal & $A 10$ \\
\hline Reino Unido & $A 6, A 7, A 21$ \\
\hline República Tcheca & $A 12$ \\
\hline Rússia & $A 8$ \\
\hline Sérvia & A15 \\
\hline Taiwan & $A 1, A 16$ \\
\hline Suécia & $A 23, A 26$ \\
\hline Suíça & $A 24$ \\
\hline
\end{tabular}

Fonte: Elaboração própria, 2017.

Nota: A coluna de "artigos vinculados" baseia-se na nacionalidade dos autores e coautores de cada estudo. 
Com base na tabela, se evidencia um maior número de estudos realizados nos Estados Unidos, Países Baixos e Reino Unido, nações que se colocam entre os 10 países mais avançados em termos de abertura de dados governamentais, em 2016, de acordo com o relatório Open Data Barometer, da World Wide Web Foundation (2016).

b) Periódicos: os estudos foram publicados em cinco diferentes periódicos especializados das áreas de Administração e Administração Pública. Dos 27 artigos, 17 foram publicados no Government Information Quarterly, seis no Information Polity, dois no Transforming Government: People, Process and Policy, um no Policy and Internet e 01 no Public Management Review. Importante ressaltar a informação de que todos os periódicos incluídos na pesquisa apresentaram na data de publicação dos estudos, fator de impacto igual ou superior à média de sua categoria, junto ao InCites Journal Citation Report (JCR), base estrangeira que avalia e compara publicações científicas utilizando dados de citações, e verifica os periódicos mais citados em uma determinada área e sua relevância para a comunidade científica;

c) Ano de publicação: dos 27 estudos publicados nos últimos cinco anos, verificou-se que sete artigos foram publicados em 2014, seis em 2015, seis em 2016 e oito artigos em 2017, o que pode significar a constância do interesse no empreendimento de pesquisas relacionadas à abertura de dados, dado o aumento de sua relevância no cenário internacional.

\subsection{Análise Temática}

A análise temática foi realizada pela leitura integral dos textos, com a geração posterior de relatórios de análise e redes semânticas. No total, foram gerados 253 códigos ou unidades de significado construídas em formato livre ou in vivo. Esses códigos resultaram em 43 subcategorias analíticas, duas categorias e um tema. As subcategorias remetem aos diferentes tipos de elementos teóricos, teorias, discussões e resultados apresentados pelos estudos, enquanto que as categorias, por sua vez, reúnem elementos e teorias, de um lado, e discussões 
e resultados, de outro, e finalmente o tema, refere-se às abordagens teóricas sobre OGD, reunindo categorias e subcategorias.

\subsubsection{Elementos e Teorias}

Nos 27 estudos analisados aparece, de forma recorrente e com relevante intensidade, referencial diretamente voltado para OGD, visando explorar conceitos, modelos, diretrizes, processos, padrões e metodologias, que dentre outras razões, são abordados com a finalidade de encorajar a publicação de dados governamentais e habilitar o uso mais fácil e acessível pelos usuários (Attard, Orlandi, Scerri \& Auer, 2015).

No que se refere às diretrizes para publicação, especialmente, se destacam as abordagens “The Five Star Scheme for Linked Open Data" (Attard et al., 2015; Chatfield \& Reddick, 2017; Vetró, Canova, Torchiano, Minotas, Iemma \& Morando, 2016; Viscusi, Spahiu, Maurino \& Batini, 2014) e "The Eight Open Government Data Principles", elaborados pelo The Open Government Working Group (Attard et al., 2015; Veljković, Bogdanović-Dinić \& Stoimenov, 2014; Fan \& Zao, 2017; Corrêa et al., 2017; Mendonça, Maciel \& Viterbo, 2015), que estabelecem critérios mínimos para disponibilização de dados governamentais, a fim de que possam ser devidamente reutilizados pelos cidadãos e demais atores que tenham interesse e desejem manipular as informações, seja para fins comerciais ou de controle e participação social, independentemente. Nesse sentido, Veljković, Bogdanović-Dinić e Stoimenov (2014) destacam que o estabelecimento de diretrizes mínimas significa que os governos devem disponibilizar dados, de maneira que seja possível sua busca e indexação, em um formato reutilizável e sem nenhuma restrição em seu manuseio.

Destaque também nos estudos para que na disponibilização de OGD sejam incluídos metadados, que consistem na descrição resumida dos dados e informações gerais, viabilizando sua busca, descoberta e localização, além de reforçar a sua autenticidade e acuracidade (Attard et al., 2015; Afful-Dadzie \& Afful-Dadzie, 2017).

No que tange às subcategorias mais voltadas diretamente a dados públicos, figura a posição recorrente dos autores sobre a necessidade da definição de um framework ou modelo padrão de disponibilização de dados dos governos, a fim de que seja proporcionada maior unicidade e respeito a determinados critérios, aceitos internacionalmente. Segundo a literatura verificada, a ausência de um framework prejudica o desenvolvimento de OGD, bem como seu 
uso efetivo e a sua conversão em benefícios para a sociedade de uma forma geral (Wang \& Lo, 2015; Fan \& Zao, 2017; Chatfield \& Reddick, 2017; Zhu, 2017).

Um outro tópico abordado refere-se às barreiras existentes relativas a OGD. Para Martin (2014), há uma perversa tendência dos governos em priorizar as questões de suprimento de dados, o que ocasiona uma falta sistemática de atenção sobre como os dados serão utilizados uma vez que estejam disponíveis. Outros autores ressaltam como barreiras as próprias características dos conjuntos de dados disponibilizados atualmente, que contém ambiguidade, incompletude e baixa qualidade (Conradie \& Choenni, 2014), resistência e aversão ao risco e à mudança, falta de legislação apropriada e políticas uniformes para a publicação de dados, falta de recursos para lançar e manter programas de dados abertos, falta de incentivos, capacidades e conhecimento técnico e contextual entre os próprios usuários e baixa qualidade dos portais eletrônicos (Dawes, Vidiasova \& Parkhimovich, 2016; Whitmore, 2014; Susha, Grönlunda \& Janssen, 2015; Wirtz, Piehler, Thomas \& Daiser, 2016). Barreiras adicionais remetem à falta de atendimento dos órgãos públicos a critérios mínimos de estrutura e configuração dos dados disponibilizados (Dawes, Vidiasova \& Parkhimovich, 2016), além do receio de parte dos governos da perda de controle face à abertura dos dados, o que figura como um inibidor institucional (Chatfield \& Reddick, 2017; Mendonça, Maciel \& Viterbo, 2015) e a dificuldade de inclusão de OGD como prioridade nas agendas públicas (Martin, 2014).

Importa ressaltar que a maioria dos estudos também destaca os potenciais benefícios com a abertura de dados governamentais, a exemplo de Dawes, Vidiasova e Parkhimovich, (2016), que evidenciam três tipos de benefícios esperados desse processo: sociais e políticos, incluindo maior transparência e accountability (prestação de contas e responsabilização), maior confiança no governo, melhoria do processo de formulação de políticas públicas e melhoria dos serviços públicos e satisfação dos cidadãos; benefícios econômicos, no sentido de encorajar inovações e possibilitar o aprimoramento de produtos, serviços e processos, gerando desenvolvimento e competitividade e, finalmente, benefícios operacionais e técnicos ao próprio governo, pelo reuso dos dados, otimização de processos administrativos e validação externa dos dados.

Inclusive, no que tange aos benefícios econômicos, a análise dos textos revela a preocupação recorrente de alguns autores em destacá-los (Zuiderwijk, Janssen, Van de Kaa \& Poulis, 2016), o que torna evidente a utilidade dos dados abertos não somente para fins políticos 
e sociais, conforme abordado inicialmente pelas pesquisas, como também pelo potencial gerador de receitas e inovações à iniciativa privada.

Nesse sentido, o potencial de alavancagem de inovações no setor privado e dentro do próprio setor público é tema que se faz presente no referencial de vários autores, que relacionam o tema de OGD ao empreendedorismo, aumento do conhecimento, desenvolvimento de atividades inovadoras e inovação aberta (Magalhaes \& Roseira, 2017; Chatfield \& Reddick, 2017; Susha, Grönlunda \& Janssen, 2015), demonstrando o importante papel que as políticas de dados abertos podem desempenhar como direcionadoras das ações de inovação nas organizações.

A respeito de teorias que foram associadas ao tema OGD merecem destaque as seguintes abordagens: Teoria Institucional, Teoria do Estado Informacional, Teoria de Ecossistemas, Teoria Cognitiva e as Teorias de Informação Interagência, da Perspectiva Multi-Nível e de Cross-boundary ou Interorganizacional, que representam uma profusão de diferentes modelos possíveis de serem utilizados, dada a amplitude da temática e os múltiplos aspectos que a compõem, levando a supor que não há neste momento predomínio de uma escola de pensamento.

Yang e Wu (2016) utilizam a Teoria Institucional no sentido de demonstrar a influência das pressões do ambiente externo para as mudanças internas nas organizações públicas, por exemplo, através da gradual institucionalização das atividades relacionadas à disponibilização de dados abertos. Para os referidos autores, as pressões advindas da mídia, usuários, órgãos de controle etc. provocam diferentes tipos de isomorfismo que impactam diretamente na intenção das organizações públicas para implementar políticas de dados abertos. Na mesma vertente, Fan e Zhao (2017) reforçam que a Teoria Institucional implica em que as normas e valores sociais e expectativas institucionais criam pressão para que as organizações públicas se adequem ou respondam de maneira aceitável a importantes constituintes sociais, alterando comportamentos e estruturas internas. Essas pressões, na opinião dos autores, direcionam o governo à partilha adicional de informações no que se refere às suas políticas, atividades e outros dados, promovendo assim uma atuação muito mais transparente.

A Teoria do Estado Informacional ou Informational State abordada por Bates (2014), por meio de uma postura mais crítica, explora a relação entre informação e poder nas instituições governamentais. Segundo seus argumentos, o estado informacional seria uma situação em que os governos deliberadamente, explicitamente e consistentemente controlam a 
criação das informações, processos e fluxos para exercer poder. Isso se traduziria na manipulação das informações disponibilizadas publicamente como ferramenta para fazer política. Seu estudo aborda o caso do Reino Unido e as intenções dos governantes daquele país em utilizarem políticas de dados abertos para reforçar os objetivos neoliberais de marketisation ou "comercialização" dos serviços públicos. Não se trata de uma posição isolada, pois essas ideias aparecem alinhadas com Martin (2014), que partilha da opinião de que o estabelecimento de uma agenda de políticas de OGD tem sido moldado por atores e instituições, a fim de levar adiante interesses capitalistas neoliberais, desvirtuando os propósitos basilares da prática de abertura de dados governamentais.

A Teoria de Ecossistemas, por sua vez, é associada ao tema OGD através da propositura de criação de um ambiente favorável ao desenvolvimento de atividades de abertura de dados e de interação constante entre os atores envolvidos. Os estudos que utilizam essa abordagem defendem que ecossistemas de dados abertos consistem tipicamente em múltiplos níveis sociotécnicos interdependentes, dimensões, atores, elementos e componentes, que se desenvolvem pela adaptação dos usuários, feedback ou retroalimentação e fornecimento dinâmico (de informações), interações entre atores e outros fatores de interação (Zuiderwijk et al., 2016; Dawes, Vidiasova \& Parkhimovich, 2016). Essa abordagem também destaca a importância do papel desenvolvido pelos governos para estimular ecossistemas de dados onde figuram desenvolvedores, inovadores e outros usuários (Dawes, Vidiasova \& Parkhimovich, 2016), promovendo assim a ampliação, difusão e consolidação da cultura de abertura de dados.

Wirtz et al. (2016), por sua vez lançam mão da Teoria Cognitiva. A preocupação é no sentido de evidenciar barreiras cognitivas ao desenvolvimento de mudanças no setor público que sejam favoráveis à abertura dos dados produzidos. Segundo os autores, fatores subjetivos e percepções individuais que levam a decisões e comportamentos podem ocasionar o enfrentamento de resistências no setor público, com as quais os gestores acabam se deparando. Assim, a Teoria Cognitiva provê bases adequadas à investigação e compreensão de questões comportamentais, modelos e processos mentais, que contemplam a aquisição, o armazenamento, a transformação e o uso de informações pelos indivíduos, em uma perspectiva psicológica. A utilização da referida abordagem pode assim se mostrar útil à superação de barreiras individuais no desenvolvimento das políticas de abertura de dados.

As últimas três teorias destacadas são as Teorias de Informação Interagência, da Perspectiva Multinível e de Cross-boundary ou Interorganizacional, apresentadas por Yang e 
Wu (2016), Martin (2014) e Fan e Zhao (2017), respectivamente. A primeira delas propõe o estudo das intenções em torno de OGD através de quatro diferentes perspectivas: cultura organizacional, benefícios, riscos percebidos e capacidades organizacionais. Para os autores, a busca pela compreensão organizacional através dessas perspectivas é de grande importância ao diagnóstico e aumento da disposição para políticas de abertura de dados governamentais.

A segunda teoria, da Perspectiva Multinível, por sua vez, advinda da área da ciência e tecnologia, serve como um insight voltado a compreender a dinâmica de mudanças transformadoras em sistemas sociotécnicos complexos. Essas mudanças transformadoras remetem à mudança de um paradigma para outro e a aplicação da teoria nesse contexto auxilia no processo de desenvolvimento das inovações - nesse caso ligadas a OGD, a fim de que se tornem sustentáveis e tenham maiores chances de sucesso. A perspectiva multinível envolve três níveis estruturais: nicho, regime sociotécnico e panorama. Cada nível representa estágios de evolução da mudança, fatores envolvidos e impactos gerados no meio, desde uma perspectiva interna até uma perspectiva externa, envolvendo toda a sociedade, sistemas e instituições.

A última teoria do conjunto, Cross-boundary ou Interorganizacional, defende uma maior interação e compartilhamento de informações e experiências entre organizações. No caso de OGD, especialmente entre organizações governamentais. O objetivo de aplicação dessa teoria é evidenciar como a partilha interorganizacional pode ser identificada como um importante direcionador para o desenvolvimento avançado de práticas de governo eletrônico, matéria que está diretamente ligada a dados governamentais. A utilização da teoria se revela importante para que, por meio de perspectivas técnicas, organizacionais, legais e políticas, estratégias sejam desenvolvidas em relação às práticas de OGD.

É possível argumentar que houve, de maneira geral, o interesse dos pesquisadores em promover a compreensão e tornar evidentes os elementos e conceitos mais intimamente ligados à abertura de dados públicos, além de elencar as diretrizes existentes e utilizadas como referência para a disponibilização desses dados, a fim de que os benefícios sejam ampliados. Por outro lado, destacam que os maiores desafios giram em torno da superação das barreiras, da estruturação de políticas que abram maior espaço nas agendas públicas, e da oferta de conjuntos de dados públicos de alta qualidade, que sejam bem descritos, bem estruturados, interconectados, de fácil acesso, primários e capazes de oferecer significado para aqueles que os manuseiam. O enfrentamento desses desafios está presente nas diferentes abordagens 
teóricas que visam congregar esforços e abranger os componentes e aspectos relevantes ao desenvolvimento do tema no setor público.

\subsection{Análise Comparativa}

Ainda com a finalidade de melhor compreender o panorama vigente de pesquisa sobre OGD, foram selecionados os estudos dos três países de origem que mais publicam sobre o tema - conforme demonstrado no tópico 3.1 da análise descritiva - para uma breve análise comparativa de seu desenho e desenvolvimento, levando-se em conta as principais referências, métodos, objetivos e resultados, o que possibilitou estabelecer um paralelo de semelhanças e divergências entre si. A síntese dos resultados dessa análise é apresentada na Tabela 2.

Conforme se verifica, não somente entre os estudos originários do mesmo país, mas entre os três países, há pontos de aproximação relevantes que podem caracterizar a forma como as pesquisas sobre a abertura de dados públicos vêm sendo conduzidas. A natureza qualitativa da maioria dos estudos e, consequentemente, a similaridade dos métodos de coleta e análise de dados representam uma dessas constatações. Contudo, a alta recorrência dos mesmos autores consultados pelos diferentes estudos, ainda que sobre temas próximos, é um achado capaz de demonstrar uma certa inter-relação entre os grupos de pesquisadores internacionalmente. 
Tabela 2: Análise comparativa: semelhanças e divergências

\begin{tabular}{|c|c|c|c|c|c|}
\hline $\begin{array}{l}\text { País de } \\
\text { Origem }\end{array}$ & Artigos & $\begin{array}{c}\text { Principais Referências } \\
\text { Teóricas }\end{array}$ & $\begin{array}{l}\text { Principais Aspectos } \\
\text { Metodológicos }\end{array}$ & Aspectos Semelhantes & $\begin{array}{c}\text { Aspectos } \\
\text { Divergentes }\end{array}$ \\
\hline $\begin{array}{l}\text { Estados } \\
\text { Unidos }\end{array}$ & $\begin{array}{c}A 2, A 3 \\
A 5, A 8, \\
A 10 \\
A 19 \\
A 27\end{array}$ & 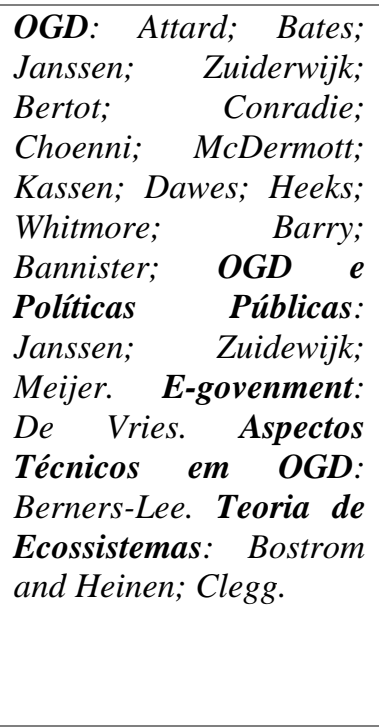 & $\begin{array}{l}\text { Desenhos de } \\
\text { Pesquisa: estudos de } \\
\text { caso. Instrumentos } \\
\text { de Coleta: portais } \\
\text { eletrônicos; } \\
\text { documentos; } \\
\text { entrevistas; } \\
\text { questionários. } \\
\text { Estratégias de } \\
\text { Análise: análise de } \\
\text { conteúdo; análise, } \\
\text { análise temática } \\
\text { histórica. }\end{array}$ & $\begin{array}{l}\text { - Predominância de } \\
\text { pesquisas de natureza } \\
\text { qualitativa; } \\
\text { - Consultas frequentes às } \\
\text { mesmas referências } \\
\text { teóricas; } \\
\text { - Aspectos } \\
\text { metodológicos bastante } \\
\text { similares; } \\
\text { - Estudos sobre políticas } \\
\text { de OGD e reutilização } \\
\text { de dados (manuseio e } \\
\text { geração de valor); } \\
\text { - Estudos sobre } \\
\text { avaliação de portais } \\
\text { eletrônicos de dados. } \\
\text { - Aplicação de modelos } \\
\text { teóricos de análise. }\end{array}$ & $\begin{array}{l}\text { - Utilização de } \\
\text { diferentes }\end{array}$ \\
\hline $\begin{array}{l}\text { Países } \\
\text { Baixos }\end{array}$ & $\begin{array}{l}A 14, \\
A 17, \\
A 20, \\
A 23\end{array}$ & $\begin{array}{l}\text { OGD: Attard; Janssen; } \\
\text { Zuiderwijk; Whitmore; } \\
\text { Lourenço; Choenni; } \\
\text { Meijer; Kassen; Barry; } \\
\text { Bannister. Teoria da } \\
\text { Informação } \\
\text { Interagência: } \quad \text { Jetzek. } \\
\text { Problemas Perversos: } \\
\text { Churchman; Ritter; } \\
\text { Weber. }\end{array}$ & $\begin{array}{lr}\text { Desenhos } & \text { de } \\
\text { Pesquisa: } & \text { revisão } \\
\text { sistemática } & \text { de } \\
\text { literatura; } & \text { estudos } \\
\text { de } & \text { caso. } \\
\text { Instrumentos } \quad \text { de } & \text { Coleta: publicações; } \\
\text { questionários; } & \\
\text { entrevistas. } & \\
\text { Estratégias } & \text { de } \\
\text { Análise: } & \text { análise } \\
\text { descritiva, } & \text { temática } \\
\text { e interpretativista. }\end{array}$ & 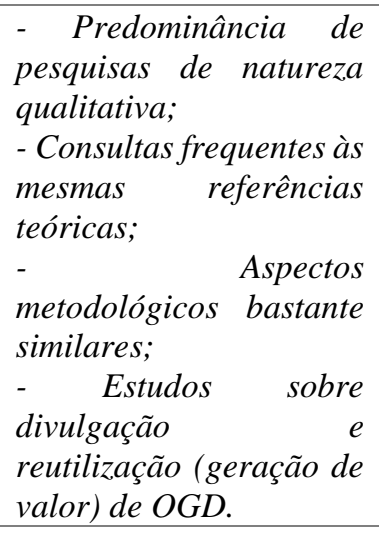 & $\begin{array}{l}\text { importados de } \\
\text { diferentes bases } \\
\text { teóricas, } \\
\text { perpassando, ex., } \\
\text { difusão da } \\
\text { inovação, } \\
\text { problemas } \\
\text { perversos, atores } \\
\text { (stakeholders), } \\
\text { ecossistemas, } \\
\text { modelos técnicos } \\
\text { (visualizaçôes, } \\
\text { web semântica), }\end{array}$ \\
\hline $\begin{array}{l}\text { Reino } \\
\text { Unido }\end{array}$ & $\begin{array}{c}A 6, A 7, \\
A 21\end{array}$ & $\begin{array}{l}\text { OGD: Bates; Janssen; } \\
\text { Zuiderwijk; Huijboom; } \\
\text { Van Den Broek; Yu; } \\
\text { Davies; Conradie and } \\
\text { Choenni. Teoria da } \\
\text { Perspectiva Multi-Nivel: } \\
\text { Geels; Rip and } \\
\text { Kemp;Geels and Schot. } \\
\text { Governo Aberto: Meijer; } \\
\text { Curtin. Nova Gestão } \\
\text { Pública: Crouch; } \\
\text { Dunleavy and Hood. } \\
\text { Neoliberalismo: Harvey; } \\
\text { Le Grand; Crouch; } \\
\text { Gamble; Hay. }\end{array}$ & $\begin{array}{l}\text { Desenhos de } \\
\text { Pesquisa: estudos de } \\
\text { caso; revisão de } \\
\text { literatura. } \\
\text { Instrumentos de } \\
\text { Coleta: entrevistas; } \\
\text { questionários; } \\
\text { observação; } \\
\text { documentos. de } \\
\text { Estratégias dére de } \\
\text { Análise: análise de } \\
\text { conteúdo; análise } \\
\text { descritiva; análise } \\
\text { de discurso. }\end{array}$ & $\begin{array}{l}\text { - Predominância de } \\
\text { pesquisas de natureza } \\
\text { qualitativa; } \\
\text { - Consultas frequentes às } \\
\text { mesmas referências } \\
\text { teóricas; } \\
\text { - Aspectos } \\
\text { metodológicos bastante } \\
\text { similares; } \\
\text { - Estudos sobre } \\
\text { barreiras e percepções } \\
\text { de atores sobre OGD; } \\
\text { - Aplicação de modelos } \\
\text { teóricos de análise. }\end{array}$ & políticas públicas. \\
\hline
\end{tabular}

Fonte: Elaboração própria, 2018. 
De maneira evidente, isso se fortalece quando levamos em conta, por exemplo, dois autores utilizados nos referenciais teóricos - Janssen e Zuiderwijk - que aparecem citados e

pelo menos 17 estudos dos 27 considerados neste trabalho, e praticamente em todos os estudos dos países que mais publicam. Além disso, boa parte dos pesquisadores em OGD referenciam-se entre si, citando as contribuições uns dos outros para o desenvolvimento do assunto, o que foi possível evidenciar na constatação de que, pelo menos oito autores dos estudos selecionados são citados por boa parte dos demais em suas publicações.

Por outro lado, quando verificados os objetivos e resultados das pesquisas, se pôde observar que há uma diversidade de propósitos que perpassam os campos teórico, técnico, social e inclusive político sobre dados governamentais. São explorados modelos teóricos de análise, além de barreiras e benefícios, utilidades, capacidades, políticas públicas, qualidade e técnica, dentre outros aspectos através dos quais se torna possível discutir o tema. Para isso, conforme já mencionado no tópico anterior, 3.2, os pesquisadores se valem de diferentes elementos teóricos e teorias associadas, que reforçam a ideia de um campo de pesquisa com múltiplas abordagens, configurando o aspecto divergente mais relevante entre as publicações.

\section{Considerações Finais}

Identificar e analisar o "estado da arte" sobre as pesquisas relacionadas a Dados Governamentais Abertos - OGD revelou-se um exercício de grande validade, na medida em que permitiu fazer um balanço sobre os diversos aspectos que demarcam a discussão, identificar quais os aportes teóricos de sustentação para exame da temática e definir como elemento central do debate a constatação de que, o apelo pela abertura das informações produzidas pelos governos vem tomando proporções mundiais nos últimos anos, devido ao fortalecimento dos mecanismos democráticos nas sociedades contemporâneas e, consequentemente, das diversas formas de controle social dos cidadãos sobre os atos de seus governantes.

Ficou claro, no entanto, que há ainda inúmeras barreiras a serem vencidas para o pleno desenvolvimento de OGD, desde elementos técnicos, políticos, culturais, organizacionais e sociais que limitam o êxito das ações empreendidas para a abertura e manutenção de dados governamentais e contribuem para o baixo nível de investimento das autoridades públicas em políticas de abertura de dados. 
Todos esses entraves implicam na necessidade de desenvolver capacidades estatais comprometidas com a abertura de espaço nas agendas públicas para o tema, seja pelos impactos sociais positivos de aproximar governos e governados, seja pelos impactos econômicos ao setor produtivo pela geração de valor e incentivo às inovações, ou ainda pelos impactos gerenciais ao setor público, pela validação dos dados abertos e melhoria de processos.

A Revisão Sistemática de Literatura contribuiu, portanto, para a compreensão da temática de OGD em diferentes países, contemplando abordagens e aspectos diversos, ao tempo em permitiu inferir que ainda não se formou uma determinada escola de pensamento na perspectiva de McKinley, Mone e Moon (2003), dado que a combinação de inovação, continuidade e escopo para a configuração do status de escola ainda não conseguiu aglutinar os estudiosos do tema de modo diferenciado.

Como limitação da pesquisa destaca-se o fato de que, unicamente nesse estudo, não foi possível discorrer sobre todo o universo de códigos mapeados, restando a decisão de abordar aqueles julgados mais recorrentes e mais relevantes ao longo de todo o período analisado.

Como sugestões de pesquisas futuras, recomenda-se explorar os estudos sobre OGD realizados no Brasil, além de estudos empíricos sobre o enfrentamento de barreiras e desafios ao desenvolvimento da matéria, análise de políticas públicas empreendidas e análises que correlacionem OGD e inovação.

\section{Referências}

Afful-Dadzie, E., \& Afful-Dadzie, A. (2017). Open Government Data in Africa: a preference elicitation analysis of media practitioners. Government Information Quarterly. Disponível em: http://dx.doi.org/10.1016/j.giq.2017.02.005.

Albano, C. S. (2014). Dados Governamentais Abertos: proposta de um modelo de produção e utilização de informações sob a ótica conceitual da cadeia de valor (Tese de Doutorado). Universidade de São Paulo, São Paulo, SP, Brasil.

Attard, J., Orlandi F., Scerri, S., \& Auer, S. (2015). A systematic review of open government data initiatives, Government Information Quarterly, 2015. Disponível em: http://dx.doi.org/10.1016/j.giq.2015.07.006.

Bates, J. (2014). The strategic importance of information policy for the contemporary neoliberal state: the case of Open Government Data in the United Kingdom. Government Information Quarterly. Disponível em: http://dx.doi.org/10.1016/j.giq.2014.02.009. 
Chatfield, A. T., \& Reddick, C. G. (2017a). A longitudinal cross-sector analysis of open data portal service capability: the case of Australian local governments. Government Information Quarterly. Disponível em: < http://dx.doi.org/10.1016/j.giq.2017.02.004>.

Chatfield, A. T., \& Reddick, C. G. (2017b). The role of policy entrepreneurs in open government data policy innovation diffusion: an analysis of Australian Federal and State Governments. Government Information Quarterly. Disponível em: http://dx.doi.org/10.1016/j.giq.2017.10.004.

Conradie, P., \& Choenni, S. (2014). On the barriers for local government releasing open data. Government Information Quarterly. Disponível em: http://dx.doi.org/10.1016/j.giq.2014.01.003.

Corrêa, A. S., Paula, E. C., Corrêa, P. L. P., \& Silva, F. S. C. (2017). Transparency and open government data: a wide national assessment of data openness in Brazilian local governments. Transforming Government: People, Process and Policy, Vol. 11, Issue: 1, pp.58-78. Disponível em: https://doi.org/10.1108/TG-12-2015-0052.

Dawes, S. S., Vidiasova, L., \& Parkhimovich, O. (2016). Planning and designing open government data programs: an ecosystem approach. Government Information Quarterly. Disponível em: http://dx.doi.org/10.1016/j.giq.2016.01.003.

Fan, B., \& Zhao, Y. (2017). The moderating effect of external pressure on the relationship between internal organizational factors and the quality of open government data. Government Information Quarterly. Disponível em: http://dx.doi.org/10.1016/j.giq.2017.08.006.

Kitchenham, B. (2007). Guidelines for performing systematic literature reviews in software engineering. V 2.3. EBSE Technical Report. Keele University: United Kingdom.

Mckinley, W., Mone, M. A., \& Moon, G. (2003). Determinantes e Desenvolvimento de Escolas na Teoria Organizacional. $R A E$, jul/set.

Magalhaes, G., \& Roseira, C. (2017). Open government data and the private sector: an empirical view on business models and value creation. Government Information Quarterly. Disponível em: http://dx.doi.org/10.1016/j.giq.2017.08.004.

Martin, C. (2014) Barriers to the Open Government Data agenda: taking a multi-level perspective. Policy \& Internet, Vol. 6, No. 3.

Mendonça, P. G. A., Maciel, C., \& Viterbo, J. (2015) Visualizing Aedes aegypti infestation in urban areas: a case study on open government data mashups. Information Polity, V 20, 119 134. Disponível em: http://dx.doi.org/10.3233/IP-150361.

Sampieri, R. H., Collado, C. F., \& Lucio, M. Del P. B. (2013). Metodologia da pesquisa. 5. ed. Porto Alegre: Penso. 
Susha, I., Grönlund, Å., \& Janssen, M. (2015). Driving factors of service innovation using open government data: an exploratory study of entrepreneurs in two countries. Information Polity, V 20, 19-34. Disponível em: http://dx.doi.org/10.3233/ip-150353.

Veljković, N., Bogdanović-Dinić, S., \& Stoimenov, L. (2014). Benchmarking open government: an open data perspective. Government Information Quarterly. Disponível em: http://dx.doi.org/10.1016/j.giq.2013.10.011.

Viscusi, G., Spahiu, B., Maurino, A., \& Batini, C. (2014). Compliance with open government data policies: an empirical assessment of Italian local public administrations. Information Polity, V 19, 263-275. Disponível em: http://dx.doi.org/10.3233/ip-140338.

Wang, H.-J., \& Lo, J. (2015). Adoption of open government data among government agencies. Government Information Quarterly. Disponível em: http://dx.doi.org/10.1016/j.giq.2015.11.004.

Whitmore, A. (2014). Using open government data to predict war: a case study of data and systems challenges. Government Information Quarterly. Disponível em: http://dx.doi.org/10.1016/j.giq.2014.04.003.

Wirtz, B. W., Piehler, R., Thomas, M.-J., \& Daiser, P. (2015). Resistance of public personnel to open government: a cognitive theory view of implementation barriers towards open government data. Public Management Review. Disponível em: < http://dx.doi.org/10.1080/14719037.2015.1103889>.

World Wide Web Foundation. (2016). Open Data Barometer. Disponível em: < http://opendatabarometer.org/? year=2016\&indicator=ODB>. Acesso em: 16 fev. 2018.

Yang, T.-M., \& Wu, Y.-J. (2016). Examining the socio-technical determinants influencing government agencies' open data publication: a study in Taiwan. Government Information Quarterly. Disponível em: http://dx.doi.org/10.1016/j.giq.2016.05.003.

Zhu, X. (2017). The failure of an early episode in the open government data movement: a historical case study. Government Information Quarterly. Disponível em: http://dx.doi.org/10.1016/j.giq.2017.03.004.

Zuiderwijk, A., Janssen, M., Kaa, G. V., \& Poulis, K. (2016). The wicked problem of commercial value creation in open data ecosystems: policy guidelines for governments. Information Polity, V 21, 223-236. Disponível em: http://dx.doi.org/ 10.3233/IP-160391. 